\title{
Finite element simulation of metal forming operations with texture based material models
}

\author{
Thomas Böhlke, Gerrit Risy and Albrecht Bertram \\ Otto-von-Guericke-Universität Magdeburg, Institut für Mechanik, PSF 4120, 39016 Magdeburg, \\ Germany \\ E-mail: boehlke@mb.uni-magdeburg.de,gerrit.risy@mb.uni-magdeburg.de and \\ bertram@mb.uni-magdeburg.de
}

Received 24 October 2005, in final form 26 January 2006

Published 9 March 2006

Online at stacks.iop.org/MSMSE/14/365

\begin{abstract}
In this paper two different texture-dependent material models based on the Taylor assumption are discussed and applied to the simulation of deep drawing operations of aluminium. From the numerical point of view, large-scale FE computations based on the Taylor model are very time-intensive and storageconsuming if the crystallographic texture is approximated by several hundred discrete crystals. Furthermore, the Taylor model in its standard form, which is based on discrete crystal orientations, has the disadvantage that the anisotropy is significantly overestimated if only a small number of crystal orientations are used. We quantitatively analyse this overestimation of anisotropy and suggest two Taylor-type models which allow us to reduce the sharpness of the crystallite orientation distribution function related to single crystals or texture components. One model is an elastic-viscoplastic Taylor model based on discrete orientations. The sharpness is reduced by modelling the isotropic background texture by an isotropic material law. The other model is a rigidviscoplastic material one, which is based on continuous model functions on the orientation space. This model allows for a direct incorporation of the scattering around an ideal texture component since the model contains the half-width as a microstructural parameter which can be biased. These models are used to compute yield stresses, $R$ values and earing profiles. The predictions are compared with experimental data.
\end{abstract}

\section{Introduction}

Ductile polycrystalline metals subjected to forming processes usually show anisotropic microstructures. A typical feature of an anisotropic microstructure is that the distributions of the grain orientation and the grain shape are strongly direction-dependent. These anisotropies induce direction-dependent yield loci and contraction ratios. Furthermore, the development of ears during deep drawing processes is commonly observed. Due to prior processing, 
the anisotropy may already exist before the forming operation; otherwise, it is induced by the mechanical process. In almost all the cases the anisotropy of the microstructure evolves during deformation. It is this evolution of anisotropy which makes the application of phenomenological material models questionable since they usually do not take into account this evolution. In order to perform reliable forming simulations, micromechanically based material models offer the opportunity to incorporate microstructural information directly into the material model and to establish a sound physical basis for the model. Taylor-type polycrystal models (Taylor 1938, van Houtte 1988, Mathur and Dawson 1989, Bronkhorst et al 1992, Miehe et al 1999) or self-consistent schemes (see, e.g. Molinari et al 1987) belong to this class of micromechanically based material models. Although computationally much more expensive than phenomenological models, they are nowadays more and more used in the integration points of finite elements in order to bridge the gap between the grain-scale and the macro-scale.

If the two-point statistics of crystal orientations is isotropic then the crystallite orientation distribution function (codf) represents the dominant aspect of the microstructure. The evolution of the codf can be modelled most easily by Taylor-type models. From the numerical point of view, large-scale computations based on the Taylor model are very time-intensive and storage-consuming if the crystallographic texture is approximated by several hundred discrete crystals. As an alternative, Raabe et al (see, e.g. 2002) or Raabe and Roters (2004) introduced the so-called texture component crystal plasticity method defined by the simplification that each texture component is described by only one discrete crystal orientation. If a texture component is modelled in such a way, the mechanical anisotropy is significantly overestimated. Raabe et al suggested that in order to reduce this overestimation, the crystal orientations used in the finite element simulation should - from integration point to integration point - scatter around the mean orientations of the texture components in order to take into account the scattering of the crystallites around the ideal components. Such an approach has the disadvantage that only the scattering is taken into account on average, i.e. if the whole sample is considered. Locally the mechanical anisotropy is still overestimated. Furthermore, it is noted that an approach based on discrete random distributions does not generally ensure a reproducibility of the numerical results. In the present work we suggest two Taylor-type models which allow for the reduction of this overestimation in different ways. One of the two models discussed here is an elastic-viscoplastic model based on discrete crystal orientations (discrete Taylor (DT) model). The model is enhanced by an isotropic constitutive equation modelling the isotropic part of the texture (discrete Taylor model with isotropic background DT(I) texture). The other model is an (elastic-)rigid-viscoplastic model based on continuous model functions on the orientation space (continuous Taylor (CT) model). The model functions permit an explicit modelling of the scattering around texture components.

The outline of the paper is as follows. In section 2 we summarize the constitutive equations of the two Taylor models used in this work. In section 3 we discuss the identification of the material parameters and texture components. In section 4 we discuss several numerical examples in detail. The first example compares the predictions of the two models DT and $\mathrm{CT}$ in the case of a model texture (rolling) which is approximated by a large number of crystal orientations by the DT model and by a small number of texture components by the CT model. In the second example we compare the predictions of the models DT and CT for an experimental cube texture which is approximated by a small number of crystal orientations by the DT and CT models. This example clearly shows that the DT model in its formulation, which is commonly used in the literature, overestimates the mechanical anisotropy by an unacceptable amount. The CT model is shown to offer a quite natural way of including the scattering around ideal texture components and, thus, of reducing the overestimation of 
anisotropy, which is an inherent property of the Taylor model. This is done by biasing the halfwidth values of the model functions describing the texture components. In the third example we introduce the aforementioned modification of the DT model which also allows for reducing the aforementioned overestimation of anisotropy. The basic idea of the modification is to describe the scattering around the texture components in the case of a discrete approximation of the codf by an isotropic material model instead of using a large number of crystal orientations. In the fourth example we analyse the yield stresses, the $R$ values and the earing profiles which are predicted by the two models for a four-component texture in an Al automotive sheet sample. The numerical results are compared with experimental data.

Notation. Throughout the text a direct tensor notation is preferred. The scalar product, the dyadic product and the Frobenius norm are denoted by $\boldsymbol{A} \cdot \boldsymbol{B}=\operatorname{tr}\left(\boldsymbol{A}^{\top} \boldsymbol{B}\right), \boldsymbol{A} \otimes \boldsymbol{B}$ and $\|\boldsymbol{A}\|=(\boldsymbol{A} \cdot \boldsymbol{A})^{1 / 2}=\left(A_{i j} A_{i j}\right)^{1 / 2}$, respectively. Symmetric and traceless tensors are designated by a prime, e.g. $\boldsymbol{A}^{\prime}$. The symmetric and the skew part of a 2 nd-order tensor $\boldsymbol{A}$ are denoted by $\operatorname{sym}(\boldsymbol{A})$ and $\operatorname{skw}(\boldsymbol{A})$, respectively. The set of proper orthogonal tensors is specified by $S O(3)$. A superimposed dot denotes the material time derivative. A superimposed bar indicates that the quantity is an effective one on the macroscale.

\section{Material models}

\subsection{Elastic-viscoplastic model}

2.1.1. Elastic law. For the formulation of the geometrically non-linear elastic-viscoplastic material model we introduce the multiplicative decomposition of the deformation gradient $\boldsymbol{F}$ into an elastic part $\boldsymbol{F}_{\mathrm{e}}$ and a plastic part $\boldsymbol{F}_{\mathrm{p}}$ (see, e.g. Mandel 1974):

$$
\boldsymbol{F}=\boldsymbol{F}_{\mathrm{e}} \boldsymbol{F}_{\mathrm{p}}
$$

Plastic deformations are assumed to be volume-preserving $\left(\operatorname{det}\left(\boldsymbol{F}_{\mathrm{p}}\right)=1\right)$. In the context of rateindependent behaviour the multiplicative decomposition can be derived based on the concept of material isomorphisms (Bertram 1999). The multiplicative decomposition is inherently non-unique. For a discussion of this issue see, for example, Bertram (2005).

The elastic strains are assumed to be small. Therefore, each linear relation between a conjugate pair of generalized stress and strain measures is applicable for the description of elastic behaviour. Here we assume a linear relation between the 2nd Piola-Kirchhoff stress tensor and Green's strain tensor in the unloaded configuration. In an Eulerian setting this assumption implies that the Kirchhoff stress tensor $\tau$ is given as a linear function of the Almansi strain tensor $\boldsymbol{E}_{\mathrm{e}}^{\mathrm{A}}$ (see, e.g. Böhlke and Bertram 2001, Böhlke 2003):

$$
\boldsymbol{\tau}=\mathbb{C}_{\mathrm{e}}\left[\boldsymbol{E}_{\mathrm{e}}^{\mathrm{A}}\right], \quad \boldsymbol{E}_{\mathrm{e}}^{\mathrm{A}}=\frac{1}{2}\left(\boldsymbol{I}-\boldsymbol{F}_{\mathrm{e}}^{-\mathrm{T}} \boldsymbol{F}_{\mathrm{e}}^{-1}\right),
$$

with $\boldsymbol{I}$ being the unit tensor. The Kirchhoff stress tensor $\tau$ is defined by the Cauchy stress tensor $\boldsymbol{\sigma}$ and the determinant $J$ of $\boldsymbol{F}$ through $\boldsymbol{\tau}=J \boldsymbol{\sigma}$. The Eulerian stiffness operator $\mathbb{C}_{\mathrm{e}}$ is given by the Rayleigh product of $\boldsymbol{F}_{\mathrm{e}}$ and the constant reference stiffness tensor $\widetilde{\mathbb{C}}$ :

$$
\mathbb{C}_{\mathrm{e}}=\boldsymbol{F}_{\mathrm{e}} \star \tilde{\mathbb{C}}=\tilde{C}_{i j k l}\left(\boldsymbol{F}_{\mathrm{e}} \boldsymbol{e}_{i}\right) \otimes\left(\boldsymbol{F}_{\mathrm{e}} \boldsymbol{e}_{j}\right) \otimes\left(\boldsymbol{F}_{\mathrm{e}} \boldsymbol{e}_{k}\right) \otimes\left(\boldsymbol{F}_{\mathrm{e}} \boldsymbol{e}_{l}\right),
$$

where $\boldsymbol{e}_{i}$ denotes the fixed sample system. Here and in the subsequent sections a tilde indicates that a quantity is formulated with respect to the unloaded configuration which is characterized by the fact that corresponding symmetry transformations are elements of $S O(3)$ (Truesdell and Noll 1965). 
The elasticity tensor can be specified by exploiting the cubic crystal symmetry of Al. In this case $\widetilde{\mathbb{C}}$ has the following projector representation (Rychlewski and Zhang 1989, Bertram and Olschewski 1993):

$$
\tilde{\mathbb{C}}=\sum_{\alpha=1}^{3} \lambda_{\alpha} \mathbb{P}_{\alpha}^{C}
$$

with the projectors

$$
\mathbb{P}_{1}^{C}=\frac{1}{3} \boldsymbol{I} \otimes \boldsymbol{I}, \quad \mathbb{P}_{2}^{C}=\mathbb{D}-\mathbb{P}_{1}^{C}, \quad \mathbb{P}_{3}^{C}=\mathbb{I}^{S}-\mathbb{P}_{2}^{C}-\mathbb{P}_{1}^{C} .
$$

$\mathbb{I}^{S}$ is the identity on symmetric 2 nd-order tensors. The anisotropic part $\mathbb{D}$ is given by a dyadic product of the lattice vectors $\tilde{\boldsymbol{g}}_{i}$,

$$
\mathbb{D}=\sum_{i=1}^{3} \tilde{\boldsymbol{g}}_{i} \otimes \tilde{\boldsymbol{g}}_{i} \otimes \tilde{\boldsymbol{g}}_{i} \otimes \tilde{\boldsymbol{g}}_{i} .
$$

The eigenvalues $\lambda_{\alpha}$ can be written in terms of components of $\tilde{\mathbb{C}}$ with respect to the orthonormal lattice vectors $\left\{\tilde{\boldsymbol{g}}_{i}\right\}(i=1,2,3): \lambda_{1}=\tilde{C}_{1111}+2 \tilde{C}_{1122}, \lambda_{2}=\tilde{C}_{1111}-\tilde{C}_{1122}$ and $\lambda_{3}=2 \tilde{C}_{1212}$. Without loss of generality, the reference lattice vectors $\tilde{\boldsymbol{g}}_{i}$ can be identified with the fixed sample system $\boldsymbol{e}_{i}$. Such a choice only affects the initial value of $\boldsymbol{F}_{\mathrm{e}}$ or $\boldsymbol{F}_{\mathrm{p}}$.

2.1.2. Flow rule. Since Al shows a rate-dependent behaviour in the room temperature range, we adopt a viscoplastic material model. The evolution equation governing $\boldsymbol{F}_{\mathrm{p}}$ is assumed to be

$$
\dot{\boldsymbol{F}}_{\mathrm{p}} \boldsymbol{F}_{\mathrm{p}}^{-1}=\tilde{\boldsymbol{k}}\left(\boldsymbol{T}_{\mathrm{e}}^{\prime}, \tau^{\mathrm{C}}\right)=\sum_{\alpha=1}^{N} \dot{\gamma}_{\alpha}\left(\boldsymbol{T}_{\mathrm{e}}^{\prime}, \tau^{\mathrm{C}}\right) \tilde{\boldsymbol{M}}_{\alpha},
$$

where $\boldsymbol{T}_{\mathrm{e}}=\boldsymbol{F}_{\mathrm{e}}{ }^{\mathrm{T}} \boldsymbol{\tau} \boldsymbol{F}_{\mathrm{e}}{ }^{-\mathrm{T}}$ denotes the Mandel stress tensor. The shear rates $\dot{\gamma}_{\alpha}$ are related to the resolved shear stresses $\tau_{\alpha}=\boldsymbol{T}_{\mathrm{e}}^{\prime} \cdot \tilde{\boldsymbol{M}}_{\alpha}$ by a power law

$$
\dot{\gamma}_{\alpha}=\dot{\gamma}_{0} \operatorname{sgn}\left(\tau_{\alpha}\right)\left|\frac{\tau_{\alpha}}{\tau^{\mathrm{C}}}\right|^{m} \text {. }
$$

Note that for the class of fcc materials it is a reasonable approximation that the slip systems harden in an isotropic manner (Kocks and Mecking 2003) such that only one critical resolved shear stress $\tau^{\mathrm{C}}$ appears in the equations. The Schmid or slip system tensors $\tilde{\boldsymbol{M}}_{\alpha}=\tilde{\boldsymbol{d}}_{\alpha} \otimes \tilde{\boldsymbol{n}}^{\alpha}$ are rank-one tensors, which are defined in terms of the slip directions $\tilde{\boldsymbol{d}}_{\alpha}$ and the slip plane normals $\tilde{\boldsymbol{n}}^{\alpha}$. In the case of an fcc single crystal at room temperature, the octahedral slip systems $\langle 1 \overline{1} 0\rangle\{111\}$ have to be taken into account $(N=12)$. The material parameter $m$ quantifies the strain rate sensitivity of the material. It is generally temperature-dependent and can be estimated by strain-rate jump experiments. The flow rule can also be equivalently formulated in terms of $\boldsymbol{F}_{\mathrm{e}}$ :

$$
\dot{\boldsymbol{F}}_{\mathrm{e}} \boldsymbol{F}_{\mathrm{e}}^{-1}=\boldsymbol{L}_{e}=\boldsymbol{L}-\boldsymbol{F}_{\mathrm{e}} \tilde{\boldsymbol{k}}\left(\boldsymbol{T}_{\mathrm{e}}^{\prime}, \tau^{\mathrm{C}}\right) \boldsymbol{F}_{\mathrm{e}}^{-1} .
$$

Here $\boldsymbol{L}=\dot{\boldsymbol{F}} \boldsymbol{F}^{-1}$ is the velocity gradient. For $\boldsymbol{F}(0)=\boldsymbol{I}$ the initial conditions of differential equations (7) and (9) are given by $\boldsymbol{F}_{\mathrm{e}}(0)=\boldsymbol{F}_{\mathrm{p}}^{-1}(0)=\boldsymbol{Q} \in S O(3)$, where $\boldsymbol{Q}=\boldsymbol{g}_{i}(0) \otimes \boldsymbol{e}_{i}$ is the initial orientation of the single crystal. $\boldsymbol{g}_{i}(0)$ are the lattice vectors at time zero.

2.1.3. Hardening law. The critical resolved shear stress $\tau^{\mathrm{C}}$ is usually related to the mean dislocation density $\rho$ in the crystal lattice by

$$
\tau^{\mathrm{C}}(\rho)=\alpha b G \sqrt{\rho},
$$


where $G$ is the shear modulus and $b$ is the magnitude of the Burgers vector. As the scalar $\alpha$ is only weakly temperature- and strain-rate-dependent, it is considered constant here. The Kocks-Mecking model describes the rate of change in the mean dislocation density in fcc single crystals over a wide range of strain rates and temperatures (Kocks 1976, Mecking 2001, Kocks and Mecking 2003). The model is given by the following set of equations:

$$
\dot{\rho}\left(\tau_{\alpha}, \rho\right)=\left(\frac{\sqrt{\rho}}{\beta b}-\kappa\left|\frac{\dot{\gamma}\left(\tau_{\alpha}, \rho\right)}{\dot{\gamma}_{0}^{*}}\right|^{-1 / n} \rho\right) \dot{\gamma}\left(\tau_{\alpha}, \rho\right),
$$

where

$$
\dot{\gamma}\left(\tau_{\alpha}, \rho\right)=\sum_{\alpha=1}^{N}\left|\dot{\gamma}_{\alpha}\left(\tau_{\alpha}, \tau^{\mathrm{C}}(\rho)\right)\right| .
$$

The first term in equation (11) describes the statistical storage of dislocations (hardening stage II). The second term in (11) models the dynamic recovery (hardening stage III), which is strain-rate- and temperature-dependent. A combination of (10) and (11) allows for deriving an equivalent formulation of the evolution equation in terms of the critical resolved shear stress

$$
\dot{\tau}^{\mathrm{C}}\left(\tau_{\alpha}, \tau^{\mathrm{C}}\right)=\Theta_{0}\left(1-\frac{\tau^{\mathrm{C}}}{\tau_{\mathrm{V}}^{\mathrm{C}}\left(\tau_{\alpha}, \tau^{\mathrm{C}}\right)}\right) \dot{\gamma}\left(\tau_{\alpha}, \tau^{\mathrm{C}}\right),
$$

where the critical Voce stress is

$$
\tau_{\mathrm{V}}^{\mathrm{C}}\left(\tau_{\alpha}, \tau^{\mathrm{C}}\right)=\tau_{\mathrm{V} 0}^{\mathrm{C}}\left|\frac{\dot{\gamma}\left(\tau_{\alpha}, \tau^{\mathrm{C}}\right)}{\dot{\gamma}_{0}^{*}}\right|^{1 / n}
$$

with $\Theta_{0}=\alpha G /(2 \beta)$ and $\tau_{\mathrm{V} 0}^{\mathrm{C}}=\alpha G /(\kappa \beta)$. The initial condition corresponding to (13) is $\tau^{\mathrm{C}}(0)=\tau_{0}^{\mathrm{C}}$.

2.1.4. Homogenization of the stresses. The relation between the macroscopic and mesoscopic stress and strain measures can be determined, e.g. based on the Taylor-type models (Taylor 1938, van Houtte 1988, Mathur and Dawson 1989, Bronkhorst et al 1992, Miehe et al 1999) or self-consistent schemes (see, e.g. Molinari et al 1987). Taylor-type models are widely used since they are computationally less expensive compared with selfconsistent schemes. Taylor-type models give a reasonable qualitative approximation of the crystallographic texture evolution in some single-phase fcc materials with high stacking-fault energy. They are known to overestimate the sharpness of the crystallographic texture and the amount of anisotropy. In the following we adopt the (Voigt-)Taylor assumption of a homogeneous deformation gradient:

$$
\boldsymbol{F}=\overline{\boldsymbol{F}} \text {. }
$$

For statistically homogeneous materials without pores and cracks, the effective Kirchhoff stress tensor is given by the volume average over the reference volume $V$. If one assumes that the crystal orientations in the grains are initially homogeneous then one obtains the following formula for the effective Kirchhoff stress tensor:

$$
\bar{\tau}=\frac{1}{V} \int_{V} \tau \mathrm{d} V=\sum_{\beta=1}^{M} v_{\beta} \tau_{\beta},
$$

where $\nu_{\beta}$ is the (initial) volume fraction of grain $\beta$ and $\tau_{\beta}$ is the corresponding Kirchhoff stress tensor. $M$ is the total number of grains, which is assumed to be finite. 


\subsection{Rigid-viscoplastic model}

2.2.1. Elastic law. In the following we adopt the assumption that dilatations are purely elastic whereas distortions are purely viscoplastic. Both deformation modes are assumed to be decoupled. Although the model contains elastic stresses related to volume changes, it is called rigid-viscoplastic. The spherical and deviatoric parts of the Kirchhoff stress tensor $\tau$ are denoted by $\tau^{\circ}$ and $\tau^{\prime}$, respectively. The first is associated with volume changes and the latter with shape changes. The spherical and deviatoric parts of the stress tensor represent the equilibrium and the dynamic part of the stress tensor, respectively (see, e.g. Šilhavý 1997). Due to the aforementioned assumptions, the strain energy density specifying the equilibrium part of the stress tensor depends only on the determinant $J$ of the deformation gradient. The following form of the strain energy density is adopted:

$$
W(J)=\frac{K}{4}\left(J^{2}-2 \ln (J)-1\right),
$$

where $K$ denotes the bulk modulus (Ogden 1972, Simo and Miehe 1992). As a result, the equilibrium stress is given by

$$
\boldsymbol{\tau}^{\circ}=J \frac{\partial W(J)}{\partial J} \boldsymbol{I}=\frac{K}{2}\left(J^{2}-1\right) \boldsymbol{I} .
$$

2.2.2. Flow rule and lattice spin. The viscoplastic distortions are assumed to result from inelastic deformations in slip systems. The slip rate is assumed to be driven by the resolved shear stress in the corresponding slip system. Therefore, it depends only on the deviatoric part of the stress tensor, which is equal to its dynamic part here. Distortions of viscoplastic single crystals can be modelled by the following set of equations:

$$
\begin{aligned}
& \boldsymbol{O}=\boldsymbol{D}^{\prime}-\boldsymbol{Q} \operatorname{sym}\left(\tilde{\boldsymbol{k}}\left(\boldsymbol{Q}^{\top} \boldsymbol{\tau}^{\prime} \boldsymbol{Q}, \tau_{\alpha}^{\mathrm{C}}\right)\right) \boldsymbol{Q}^{\top}, \\
& \dot{\boldsymbol{Q}} \boldsymbol{Q}^{-1}=\boldsymbol{W}-\boldsymbol{Q} \operatorname{skw}\left(\tilde{\boldsymbol{k}}\left(\boldsymbol{Q}^{\top} \boldsymbol{\tau}^{\prime} \boldsymbol{Q}, \tau_{\alpha}^{\mathrm{C}}\right)\right) \boldsymbol{Q}^{\top},
\end{aligned}
$$

(see, e.g. Hutchinson (1976)). $\boldsymbol{D}^{\prime}$ and $\boldsymbol{W}$ are the traceless symmetric and the skew part of the velocity gradient $\boldsymbol{L}$. An orthogonal tensor $\boldsymbol{Q}$ is used in order to specify the single crystal orientation. $\boldsymbol{Q}$ is introduced in such a way that it maps a reference basis $\boldsymbol{e}_{i}$ onto the lattice vectors $\boldsymbol{g}_{i}$ at a time $t \geqslant 0: \boldsymbol{g}_{i}(t)=\boldsymbol{Q}(t) \boldsymbol{e}_{i}$. If $\boldsymbol{g}_{i}(t)$ is known, the orthogonal tensor $\boldsymbol{Q}$ can be computed by $\boldsymbol{Q}=\boldsymbol{g}_{i}(t) \otimes \boldsymbol{e}_{i}$. For a given strain rate tensor $\boldsymbol{D}^{\prime}$ and a crystal orientation $\boldsymbol{Q}$, equation (19) $)_{1}$ is an implicit equation for the stress deviator $\boldsymbol{\tau}^{\prime}$. For given $\boldsymbol{\tau}^{\prime}, \boldsymbol{W}$ and $\boldsymbol{Q}$, respectively, equation $(19)_{2}$ determines the spin $\dot{Q} Q^{-1}$ of the crystal lattice. Note that equations (19) can be derived from equation (9). The tensor $\boldsymbol{Q}$ corresponds to the orthogonal part $\boldsymbol{R}_{e}$ of $\boldsymbol{F}_{\mathrm{e}}$.

The function $\tilde{\boldsymbol{k}}$ is assumed to be given by

$$
\tilde{\boldsymbol{k}}\left(\boldsymbol{Q}^{\top} \boldsymbol{\tau}^{\prime} \boldsymbol{Q}, \tau^{\mathrm{C}}\right)=\sum_{\alpha=1}^{N} \dot{\gamma}_{\alpha}\left(\tau_{\alpha}, \tau^{\mathrm{C}}\right) \tilde{\boldsymbol{M}}_{\alpha},
$$

with

$$
\dot{\gamma}_{\alpha}\left(\tau_{\alpha}, \tau^{\mathrm{C}}\right)=\dot{\gamma}_{0} \operatorname{sgn} \tau_{\alpha}\left|\frac{\tau_{\alpha}}{\tau^{\mathrm{C}}}\right|^{m}
$$

and

$$
\tau_{\alpha}=\left(\boldsymbol{Q}^{\top} \boldsymbol{\tau}^{\prime} \boldsymbol{Q}\right) \cdot \tilde{\boldsymbol{M}}_{\alpha}
$$

(Hutchinson 1976). In the limit $m \rightarrow \infty$ a rate-independent behaviour is obtained. Equation (20) implies that the rate of deformation is positively homogeneous of degree $m$ in the stress tensor, whereas the stress is homogeneous of degree $1 / m$ in the rate of deformation. The hardening model is the same as in the elastic-viscoplastic model. 
2.2.3. Homogenization of the stresses. It is assumed that the material parameters are homogeneous in the aggregate. The stress field in the polycrystal can be computed if the fields $J, \boldsymbol{D}^{\prime}, \boldsymbol{Q}$ and $\tau^{\mathrm{C}}$ are known. In the following we adopt the (Voigt-)Taylor assumption that the polycrystal deforms homogeneously on the microscale: $\bar{J}=J, \overline{\boldsymbol{D}}^{\prime}=\boldsymbol{D}^{\prime}$. Within this upper bound approximation, macroscopic anisotropies exist due to an inhomogeneous distribution of the crystal orientations $Q \in S O(3)$ and the hardening state $\tau^{\mathrm{C}} \in \mathbb{R}^{+}$.

The state of the polycrystal will be described by a distribution function $h\left(\boldsymbol{Q}, \tau^{\mathrm{C}}\right)$ :

$$
\frac{\mathrm{d} V}{V}\left(\boldsymbol{Q}, \tau^{\mathrm{C}}\right)=h\left(\boldsymbol{Q}, \tau^{\mathrm{C}}\right) \mathrm{d} Q \mathrm{~d} \tau^{\mathrm{C}},
$$

which specifies the volume fraction of crystals having the orientation $\boldsymbol{Q}$ and the drag stress $\tau^{\mathrm{C}}$. The distribution function $h\left(\boldsymbol{Q}, \tau^{\mathrm{C}}\right)$ is non-negative

$$
h\left(\boldsymbol{Q}, \tau^{\mathrm{C}}\right) \geqslant 0 \quad \forall \boldsymbol{Q} \in S O(3), \quad \tau^{\mathrm{C}} \in \mathbb{R}^{+}
$$

and normalized by

$$
\int_{\mathcal{H}} h\left(\boldsymbol{Q}, \tau^{\mathrm{C}}\right) \mathrm{d} Q \mathrm{~d} \tau^{\mathrm{C}}=1
$$

where $\mathcal{H}=\mathbb{R}^{+} \times S O(3)$. If $S O(3)$ is parametrized by Euler angles, the volume element $\mathrm{d} Q$ is given by

$$
\mathrm{d} Q=\frac{\sin (\Phi)}{8 \pi^{2}} \mathrm{~d} \varphi_{1} \mathrm{~d} \Phi \mathrm{d} \varphi_{2}
$$

The codf $f(\boldsymbol{Q})$ (Bunge 1993, Böhlke 2005) is obtained by integrating $h\left(\boldsymbol{Q}, \tau^{\mathrm{C}}\right)$ over $\mathbb{R}^{+}$

$$
f(\boldsymbol{Q})=\int_{\mathbb{R}^{+}} h\left(\boldsymbol{Q}, \tau^{\mathrm{C}}\right) \mathrm{d} \tau^{\mathrm{C}}
$$

The effective stress tensor is given by the average of the Kirchhoff stress tensor over the reference volume $V$. Based on the Taylor assumption and by means of the distribution function $h$, the macroscopic stress tensor can be computed by

$$
\bar{\tau}=\frac{1}{V} \int_{V}\left(\tau^{\circ}+\tau^{\prime}\right) \mathrm{d} V=\tau^{\circ}+\tau^{\prime}
$$

where

$$
\overline{\boldsymbol{\tau}}^{\circ}=\bar{J} \frac{\partial W(\bar{J})}{\partial \bar{J}} \boldsymbol{I}=\frac{K}{2}\left(J^{2}-1\right) \boldsymbol{I}
$$

and

$$
\overline{\boldsymbol{\tau}}^{\prime}=\int_{\mathcal{H}} h\left(\boldsymbol{Q}, \tau^{\mathrm{C}}\right) \boldsymbol{\tau}^{\prime}\left(\overline{\boldsymbol{D}}^{\prime}, \boldsymbol{Q}, \tau^{\mathrm{C}}\right) \mathrm{d} Q \mathrm{~d} \tau^{\mathrm{C}} .
$$

If the hardening state is homogeneous in the aggregate, i.e. $h\left(\boldsymbol{Q}, \tau^{\mathrm{C}}\right) \sim f(\boldsymbol{Q}) \delta_{\tau^{\mathrm{c}}}$, then the last equation can be simplified to an orientational average of the stress tensor

$$
\overline{\boldsymbol{\tau}}^{\prime}=\int_{S O(3)} f(\boldsymbol{Q}) \boldsymbol{\tau}^{\prime}\left(\overline{\boldsymbol{D}}^{\prime}, \boldsymbol{Q}, \tau^{\mathrm{C}}\right) \mathrm{d} Q .
$$

2.2.4. Texture components. Crystallographic textures can often be described by a small number of texture components or texture fibres (Wasserman and Grewen 1962, Bunge 1993, Kocks et al 1998). A texture component is a crystal orientation for which the codf shows a (local) maximum in the elementary region. In its neighbourhood, the codf decreases in an isotropic or anisotropic way. A commonly used model function, which describes a central distribution, is the Mises-Fischer distribution. It has the maximum entropy of 
all orientation distributions on $S O(3)$ with the expectation value of $\boldsymbol{Q}$ equal to $\boldsymbol{Q}_{\alpha}$. This distribution function was introduced by von Mises in the two-dimensional case and by Fischer in the three-dimensional case (Mardia and Jupp 2000). Matthies (1980) was the first to apply the Mises-Fischer distribution in texture analysis. He called it a normal distribution in the orientation space (see also Matthies et al 1988), but this interpretation was criticized by Schaeben (1992, 1994). Eschner (1993) and Eschner and Fundenberger (1997) used noncentral distribution functions for the description of experimental crystallographic textures. An overview on central and non-central distribution functions on $S O(3)$ can be found in the monograph by Mardia and Jupp (2000).

The distribution function $h\left(\boldsymbol{Q}, \tau^{\mathrm{C}}\right)$ is decomposed into an isotropic part $h^{\mathrm{I}}$ and an anisotropic part $h^{\mathrm{A}}$ with volume fractions $v^{\mathrm{I}}$ and $v^{\mathrm{A}}$, respectively. The anisotropic part is modelled by a superposition of $N_{c}$ central distributions $g\left(\boldsymbol{Q}, \boldsymbol{Q}_{\alpha}, b_{\alpha}\right)$ with mean orientations $\boldsymbol{Q}_{\alpha}$ and volume fractions $v_{\alpha}^{\mathrm{A}}\left(\alpha=1, \ldots, N_{c}\right)$. The hardening state of each of the components is assumed to be homogeneous and hence can be specified in terms of one drag stress $\tau_{\alpha}^{\mathrm{C}}$. Also by assumption the isotropic part depends on the arithmetic mean of the drag stresses $\bar{\tau}^{\mathrm{C}}=\sum_{\alpha=1}^{N_{c}} v_{\alpha}^{\mathrm{A}} \tau_{\alpha}^{\mathrm{C}}$. As a result, we have

$$
h\left(\boldsymbol{Q}, \tau^{\mathrm{C}}\right)=v^{\mathrm{I}} h^{\mathrm{I}}\left(\bar{\tau}^{\mathrm{C}}\right)+v^{\mathrm{A}} h^{\mathrm{A}}\left(\boldsymbol{Q}, \boldsymbol{Q}_{\alpha}, b_{\alpha}, \tau_{\alpha}^{\mathrm{C}}\right),
$$

where

$$
h^{\mathrm{I}}\left(\bar{\tau}^{\mathrm{C}}\right) \sim \delta_{\bar{\tau}^{\mathrm{C}}}
$$

and

$$
h^{\mathrm{A}}\left(\boldsymbol{Q}, \boldsymbol{Q}_{\alpha}, b_{\alpha}, \tau_{\alpha}^{\mathrm{C}}\right) \sim \sum_{\alpha=1}^{N_{c}} v_{\alpha}^{\mathrm{A}} g\left(\boldsymbol{Q}, \boldsymbol{Q}_{\alpha}, b_{\alpha}\right) \delta_{\tau_{\alpha}^{\mathrm{C}}} .
$$

The following constraints upon the volume fractions hold:

$$
v^{\mathrm{I}}+v^{\mathrm{A}}=1 \quad \sum_{\alpha=1}^{N_{c}} v_{\alpha}^{\mathrm{A}}=1 .
$$

The value of a central distribution $g\left(\boldsymbol{Q}, \boldsymbol{Q}_{\alpha}, b_{\alpha}\right)$ at $\boldsymbol{Q}$ depends only on the distance $\omega$ between $\boldsymbol{Q}$ and $\boldsymbol{Q}_{\alpha}$, which is generally given by

$$
\omega\left(\boldsymbol{Q}, \boldsymbol{Q}_{\alpha}\right)=\arccos \left(\frac{1}{2}\left(\operatorname{tr}\left(\boldsymbol{Q} \boldsymbol{Q}_{\alpha}^{-1}\right)-1\right)\right)
$$

(Bunge 1993). A specific central distribution is the Mises-Fischer distribution

$$
g\left(\boldsymbol{Q}, \boldsymbol{Q}_{\alpha}, b_{\alpha}\right)=N\left(S_{\alpha}\right) \mathrm{e}^{\left(S_{\alpha} \cos \left(\omega\left(\boldsymbol{Q}, \boldsymbol{Q}_{\alpha}\right)\right)\right)},
$$

where

$$
N_{\alpha}=\frac{1}{I_{0}\left(S_{\alpha}\right)-I_{1}\left(S_{\alpha}\right)}
$$

and

$$
S_{\alpha}=S\left(b_{\alpha}\right)=\frac{\ln (2)}{2 \sin ^{2}\left(b_{\alpha} / 4\right)} .
$$

The parameter $b_{\alpha}$ represents the half-width of the distribution. The modified Bessel functions $I_{n}$ are defined by

$$
I_{n}(S)=\frac{1}{\pi} \int_{0}^{\pi} \exp (S \cos (t)) \cos (n t) \mathrm{d} t .
$$


A distribution function $h$ reflects both the symmetry of the crystallites forming the aggregate and the sample symmetry, which results from the processing history (Zheng and Fu 2001). The crystal symmetry implies the following symmetry relation:

$$
h\left(\boldsymbol{Q}, \tau^{\mathrm{C}}\right)=h\left(\boldsymbol{Q} \boldsymbol{H}^{\mathrm{C}}, \tau^{\mathrm{C}}\right)
$$

$\forall \boldsymbol{H}^{\mathrm{C}} \in S^{\mathrm{C}} \subseteq S O(3) . S^{\mathrm{C}}$ denotes the symmetry group of the single crystal, which is assumed here to have a cubic symmetry. The sample symmetry implies the following symmetry relation:

$$
h\left(\boldsymbol{Q}, \tau^{\mathrm{C}}\right)=h\left(\boldsymbol{H}^{\mathrm{S}} \boldsymbol{Q}, \tau^{\mathrm{C}}\right)
$$

$\forall \boldsymbol{H}^{\mathrm{S}} \in S^{\mathrm{S}} \subseteq S O(3) . S^{\mathrm{S}}$ denotes the symmetry group of the sample. The following modified Mises-Fischer distribution implies the fulfilment of constraint (41) upon the function $h$

$$
g\left(\boldsymbol{Q}, \boldsymbol{Q}_{\alpha}, b_{\alpha}\right)=\frac{1}{24} \sum_{\beta=1}^{24} N_{\alpha} \mathrm{e}^{S_{\alpha} \cos \left(\omega\left(\boldsymbol{Q H} \boldsymbol{H}_{\beta}^{\mathrm{C}} \boldsymbol{Q}_{\alpha}^{-1}\right)\right)},
$$

where the $\boldsymbol{H}_{\alpha}^{\mathrm{C}} \in S O(3)$ are the 24 elements of the symmetry group of cubic crystals.

Up to now the anisotropic part of the stress deviator is given by the orientational average

$$
\overline{\boldsymbol{\tau}}^{\prime A}=\int_{S O(3)} \sum_{\alpha=1}^{N_{c}} v^{\mathrm{A}} v_{\alpha}^{\mathrm{A}} g\left(\boldsymbol{Q}, \boldsymbol{Q}_{\alpha}, b_{\alpha}\right) \boldsymbol{\tau}^{\prime}\left(\overline{\boldsymbol{D}}^{\prime}, \boldsymbol{Q}, \tau_{\alpha}^{\mathrm{C}}\right) \mathrm{d} Q .
$$

After defining $\xi_{\alpha}$ by $\tau_{\alpha}^{\mathrm{C}}=\xi_{\alpha} \bar{\tau}^{\mathrm{C}}$ and taking into account that the stress deviator is homogeneous of degree $1 / \mathrm{m}$ in the rate of deformation, it is possible to reformulate equation (44) by

$$
\overline{\boldsymbol{\tau}}^{\prime A}=\int_{S O(3)} f^{A}(\boldsymbol{Q}) \boldsymbol{\tau}^{\prime}\left(\overline{\boldsymbol{D}}, \boldsymbol{Q}, \bar{\tau}^{\mathrm{C}}\right) \mathrm{d} Q
$$

where

$$
f^{A}(\boldsymbol{Q})=\sum_{\alpha=1}^{N_{c}} \hat{v}_{\alpha}^{\mathrm{A}} g\left(\boldsymbol{Q}, \boldsymbol{Q}_{\alpha}, b_{\alpha}\right)
$$

and $\hat{v}_{\alpha}^{\mathrm{A}}=v^{\mathrm{A}} v_{\alpha}^{\mathrm{A}} \xi_{\alpha}^{m}$. It can be seen that an inhomogeneous hardening state affects the volume fractions which occur in the orientation distribution function. Since the stress deviator is implicitly given, the last equation makes the numerical determination of the stress tensor easier. For a given $\boldsymbol{D}^{\prime}$ the implicit equation has to be solved only once at $\bar{\tau}^{\mathrm{C}}$.

\section{Parameter identification and numerical implementation}

\subsection{Material parameters}

The material parameters of $\mathrm{Al}$ have been determined based on experimental data documented in the literature and are given in table 1. The elastic constants are taken from Brandes and Brook (1998). The strain rate sensitivity parameter $m$ has been determined by Les et al (1999). The hardening parameters are computed using experimental data documented by Mecking (2001). The material parameters of the rigid-viscoplastic model that occur in the flow rule and the hardening rule coincide with the parameters of the elastoviscoplastic model. Due to neglection of elastic distortion only one elastic constant has to be specified. The bulk modulus is taken as $K=77.3 \mathrm{GPa}$ which corresponds to the elastic constants of the single crystals. In subsequent examples we will always use identical parameter sets which qualitatively reflect the main features of the mechanical behaviour of Aluminium at room temperature. This simplification is introduced since in the referred literature only limited experimental data are given. 
Table 1. Material parameters of Al.

\begin{tabular}{llll}
\hline Elastic law & $\tilde{C}_{1111}$ & {$[\mathrm{GPa}]$} & 108 \\
& $\tilde{C}_{1122}$ & {$[\mathrm{GPa}]$} & 62 \\
& $\tilde{C}_{1212}$ & {$[\mathrm{GPa}]$} & 28.3 \\
Flow rule & $\dot{\gamma}_{0}$ & {$\left[\mathrm{~s}^{-1}\right]$} & $9 \cdot 10^{-3}$ \\
& $m$ & {$[-]$} & 133 \\
& $\tilde{\boldsymbol{M}}_{\alpha}$ & {$[-]$} & $\{111\}\langle 110\rangle$ \\
Hardening rule & $\Theta_{0}$ & {$[\mathrm{MPa}]$} & 37.5 \\
& $\tau_{\mathrm{V} 0}^{\mathrm{C}}$ & {$[\mathrm{MPa}]$} & 51.5 \\
& $n$ & {$[-]$} & 22.5 \\
& $\dot{\gamma}_{0}^{*}$ & {$\left[\mathrm{~s}^{-1}\right]$} & $10^{7}$ \\
& $\tau_{0}^{\mathrm{C}}$ & {$[\mathrm{MPa}]$} & 15 \\
\hline
\end{tabular}

\subsection{Texture components}

If the codf of a polycrystal is known then it is possible to calculate the main components of the texture of the polycrystal. The texture (reference texture) results from a simulation or an experiment. The calculated components can be used as the start values for the introduced component model. The identification procedure of the components is based on the comparison of the reference texture and a model texture. Here, the model texture consists of a fixed number of texture components characterized by Mises-Fischer distributions. The comparison of both textures can be performed by defining a distance between the related codfs:

$$
\delta=\int_{S O(3)}\left(f_{\text {ref }}(\boldsymbol{Q})-f(\boldsymbol{Q})\right)^{2} \mathrm{~d} Q
$$

where $f_{\text {ref }}(\boldsymbol{Q})$ describes the reference codf and $f(\boldsymbol{Q})$ defines a model codf consisting of $N_{c}$ components. The $N_{c}$ components can be computed by minimizing the distance $\delta$

$$
\delta\left(\boldsymbol{x}\left(\boldsymbol{Q}_{1}, \ldots, \boldsymbol{Q}_{N_{c}}\right), b_{1}, \ldots, b_{N_{c}}, v_{1}, \ldots, v_{N_{c}}, f_{\text {ref }}\right) \rightarrow \min .
$$

The list $\boldsymbol{x}$ in equation (48) contains the Euler angles defining the mean orientations of the components and has dimension $3 N_{c}$ :

$$
\boldsymbol{x}=\left[\varphi_{1}^{1}, \Phi^{1}, \varphi_{2}^{1}, \ldots, \varphi_{1}^{N_{c}}, \Phi^{N_{c}}, \varphi_{2}^{N_{c}}\right] .
$$

It is assumed that these Euler angles are variable during the optimization process, while the volume fractions $v_{i}$ and the half-widths $b_{i}$ of the components are kept constant. Because of periodicity of the Euler space, the optimization problem is unconstrained. The objective function has several local minima, but it is of importance to find the global minimum. Therefore an optimization method is applied (Boender et al 1982, Csendes et al 1986) which is able to find the global optimum with high probability. In this method a stochastic algorithm is used which is based on the development of clusters for isolating the global optimum. For the local search procedure, a quasi-Newton method is applied.

\subsection{Numerical implementation}

Both material models have been implemented into the finite element code ABAQUS (ABAQUS/Standard 2003) using the interface UMAT. In the case of the elastic-viscoplastic material model the internal variables are updated in time using the Euler backward scheme in an incrementally objective setting. The corresponding algorithmic linearization has been implemented. The constraint of the plastic incompressibility is ensured by a projection method. 


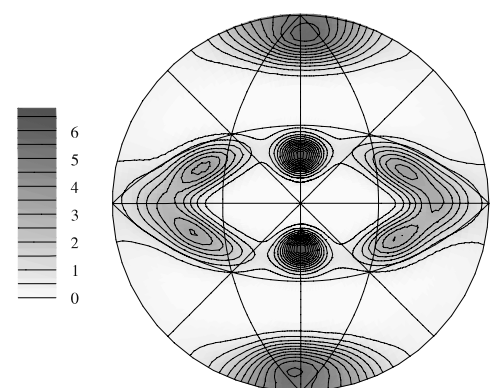

(a) 1000 crystals

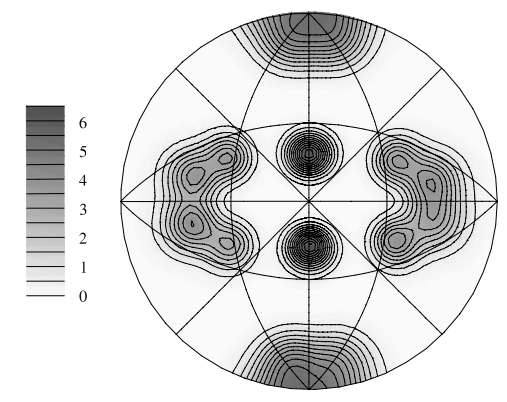

(b) 4 components

Figure 1. $\{111\}$ Pole figure of a rolling texture predicted by the Taylor model $(a)$. Approximation of the codf by four texture components $(b)$ (horizontal axis: transverse direction, vertical axis: rolling direction).

In the case of the rigid-viscoplastic material model, the strain rate determines the stress in an implicit fashion. The equation is solved based on an incrementally objective estimate of the rate of deformation tensor. After having determined this stress tensor, the lattice rotation is updated with an explicit exponential map. The numerical integration over the orientation space is performed by a Gauss point quadrature. Before applying this scheme, the orientation space has been transformed such that the metric is position-independent.

\section{Numerical examples}

\subsection{Example: rolling texture}

In this example we compare the Taylor-type model which is based on discrete crystal orientations with that formulated by means of continuous model functions for the special case where the DT model uses a large number of crystal orientations. The aim is to predict the final earing profile, the yield stress and the $R$ value based on the initial texture.

The initial texture is a model texture given by a Taylor simulation (DT) of a rolling deformation with 1000 crystals. The thickness reduction is $90 \%$. The $\{111\}$ pole figure is shown in figure 1 $(a)$. The discrete codf given by the Taylor simulation is taken as the reference codf for the identification of texture components. The continuous reference codf has been estimated by a superposition of 1000 Mises-Fischer distributions, one for each single crystal, with a half-width of $15^{\circ}$. The identification procedure of texture components has been described in section 3.2. Four texture components are used to approximate the rolling texture (CT). The corresponding $\{111\}$ pole figure is shown in figure $1(b)$. The half-width values, Euler angles and volume fractions which are the result of the optimization procedure are given in table 2 .

In the continuous model a texture component is described by three orientation variables and one hardening variable. In the case of the DT model, one single crystal is described by eight variables specifying the lattice strain and lattice orientation and one hardening variable. As a result, the continuous model has the advantage that a much smaller number of internal variables is involved ( $4 \times 4=16$ versus $1000 \times 9=9000$ in each integration point). One finds that the calculation times of the CT model are approximately eight times shorter than the corresponding calculation times of the DT model. Of course, due to the numerical integration over the orientation space the computational effort of the continuous model with four components would be larger than the one for the DT model with the same number of crystals. 
Table 2. Half-width, volume fraction and Euler angles of four Mises-Fischer model functions approximating the rolling texture.

\begin{tabular}{lllrrr}
\hline$i$ & $b_{i}$ & $v_{i}$ & \multicolumn{1}{c}{$\varphi_{1}^{i}$} & \multicolumn{1}{c}{$\Phi^{i}$} & \multicolumn{1}{c}{$\varphi_{2}^{i}$} \\
\hline 1 & $17.8^{\circ}$ & 0.25 & $67.27^{\circ}$ & $29.05^{\circ}$ & $-28.32^{\circ}$ \\
2 & $17.8^{\circ}$ & 0.25 & $-74.54^{\circ}$ & $-28.13^{\circ}$ & $33.55^{\circ}$ \\
3 & $17.9^{\circ}$ & 0.27 & $73.12^{\circ}$ & $-28.25^{\circ}$ & $-32.53^{\circ}$ \\
4 & $18.5^{\circ}$ & 0.23 & $-65.75^{\circ}$ & $29.34^{\circ}$ & $27.18^{\circ}$ \\
\hline
\end{tabular}
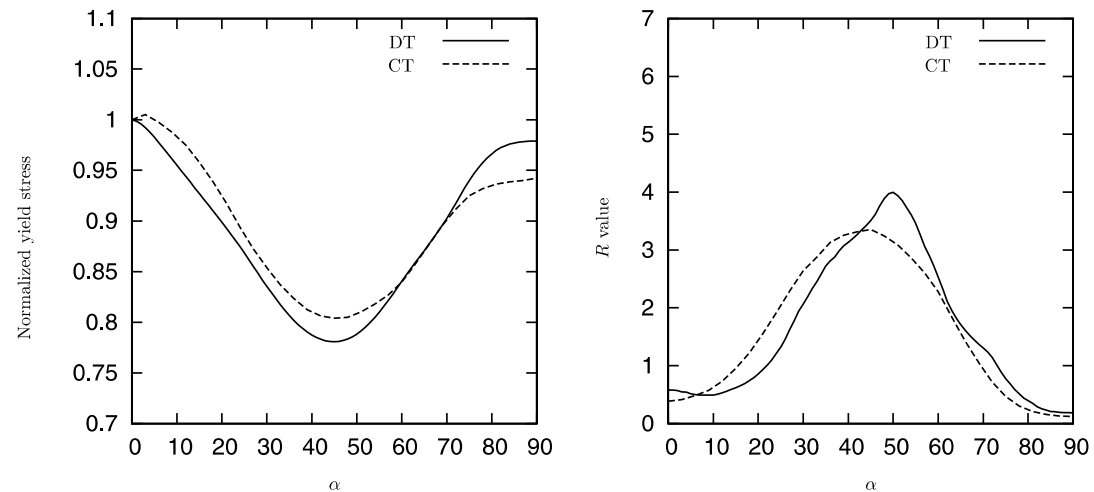

Figure 2. Normalized yield stresses (left) and $R$ values (right) versus the angle to the rolling direction in the sheet plane for the Taylor-type simulations.

The geometrical parameters for the simulation of the deep drawing process are as follows: blank diameter $d_{\mathrm{b}}=60 \mathrm{~mm}$, punch diameter $d_{\mathrm{p}}=33.3 \mathrm{~mm}$, thickness $t_{\mathrm{b}}=0.5 \mathrm{~mm}$, punch profile radius $r_{\mathrm{pp}}=6 \mathrm{~mm}$ (see also figure 5). Because of orthotropic sample symmetry of the codf it is possible to model only a quarter of the sheet. The quarter of the sheet has been discretized by $960 \mathrm{C} 3 \mathrm{D} 8 \mathrm{H}$ and $132 \mathrm{C} 3 \mathrm{D} 6 \mathrm{H}$ elements. The application of shell elements would give more accurate results but the aforementioned elements are used for simplicity in this large strain anisotropic plasticity problem.

In figure 2 the yield stress and the $R$ value in the initial state are shown for both models. The yield stress and the $R$ value are computed by performing tensile tests with one finite element. Figure 3 shows the normalized and the total earing profile predicted by the two models. The numerical results indicate that both models predict almost identical yield stresses and $R$ values. This is worth mentioning since one model neglects elastic distortions completely. Also the earing profiles are quite similar indicating that here also the elastic distortions are of minor importance. In fact, the viscoplastic model is generally unable to predict spring-back phenomena. Such effects, however, are not considered in this paper.

\subsection{Example: cube texture}

In the second example we compare the predictions of the two Taylor-type models for a texture which can be described by a small number of texture components. Engler and Kalz (2004) determined the crystallographic texture of an aluminium sheet and the corresponding earing profile resulting from a deep drawing process. The crystallographic texture has been determined by measuring pole figures in back reflection by means of a standard x-ray texture goniometer. The codf has been calculated by the series expansion method $\left(l_{\max }=22\right)$ under the assumption of an orthotropic sample symmetry. The texture is dominated by 

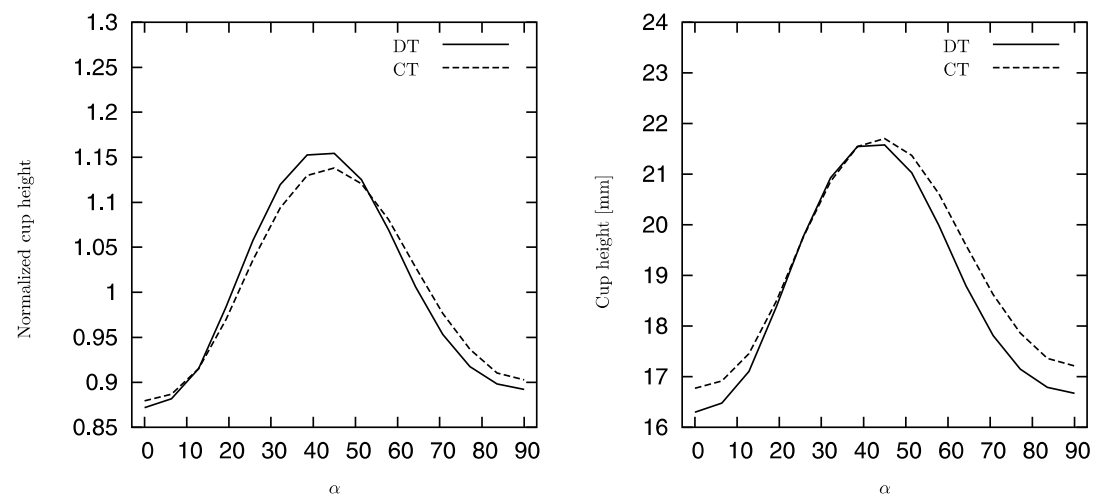

Figure 3. Normalized and absolute earing profiles for the Taylor-type texture shown in figure 1 calculated by the classical Taylor model and the component model.

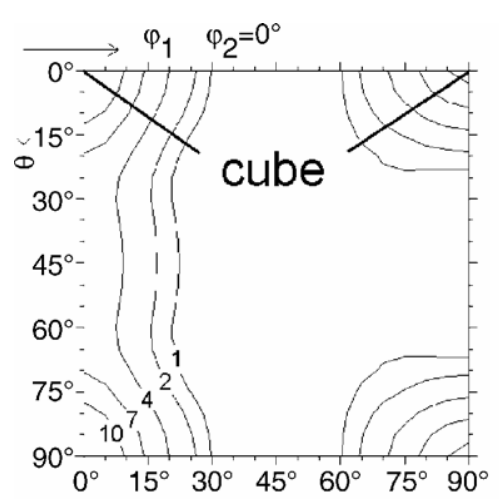

(a) Experiment

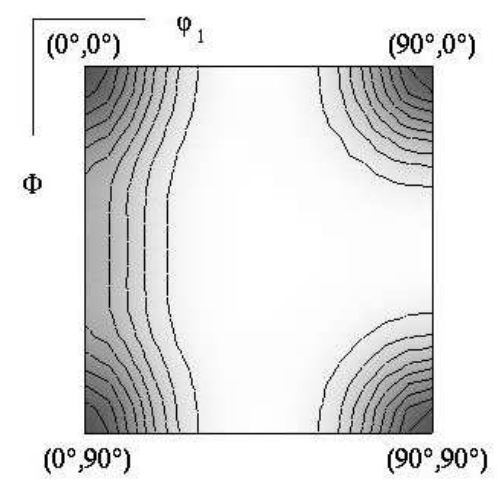

(b) Simulation

Figure 4. Section of the orientation space showing the experimental cube texture with a goss component and an approximation by texture components.

a cube component $\left(\varphi_{1}=0^{\circ}, \Phi=0^{\circ}, \varphi_{2}=0^{\circ}\right)$ but also shows a weak goss component $\left(\varphi_{1}=0^{\circ}, \Phi=45^{\circ}, \varphi_{2}=0^{\circ}\right)$. Figure 4 shows the experimental codf in a section of the orientation space $\left(\varphi_{2}=0^{\circ}\right)$.

In order to specify the initial texture for the continuous model (CT) the texture is approximated by a set of six texture components (Mises-Fischer model functions), the parameters of which are given in table 3. Both the cube and goss components are approximated by one Mises-Fischer model function. Beside these two components four other components are used for the approximation. These orientations are obtained by rotating the cube orientation about the rolling direction by $15^{\circ}, 30^{\circ}, 60^{\circ}, 75^{\circ}$, respectively. For the discrete model (DT) the midpoints of the texture components are used for specification of the initial texture. The geometrical parameters have been taken from Engler and Kalz (2004) which have also been used in the last example (figure 5). The discretization of the blank is identical to this example. The deformed mesh is shown in figure 6.

The predictions of the DT and CT models for the earing profile are shown in figure 7 together with the experimental data by Engler and Kalz (2004). In the paper by 
Table 3. Half-widths, volume fractions and Euler angles, respectively, for the Mises-Fischer components approximating the texture shown in figure 4.

\begin{tabular}{llllll}
\hline$i$ & $b_{i}$ & $v_{i}$ & $\varphi_{1}^{i}$ & $\Phi^{i}$ & $\varphi_{2}^{i}$ \\
\hline 1 (cube) & $32.5^{\circ}$ & 0.6 & 0 & 0 & 0 \\
2 & $27.5^{\circ}$ & 0.06 & 0 & $15^{\circ}$ & 0 \\
3 & $27.5^{\circ}$ & 0.08 & 0 & $30^{\circ}$ & 0 \\
4 (goss) & $27.5^{\circ}$ & 0.12 & 0 & $45^{\circ}$ & 0 \\
5 & $27.5^{\circ}$ & 0.08 & 0 & $60^{\circ}$ & 0 \\
6 & $27.5^{\circ}$ & 0.06 & 0 & $75^{\circ}$ & 0 \\
\hline
\end{tabular}

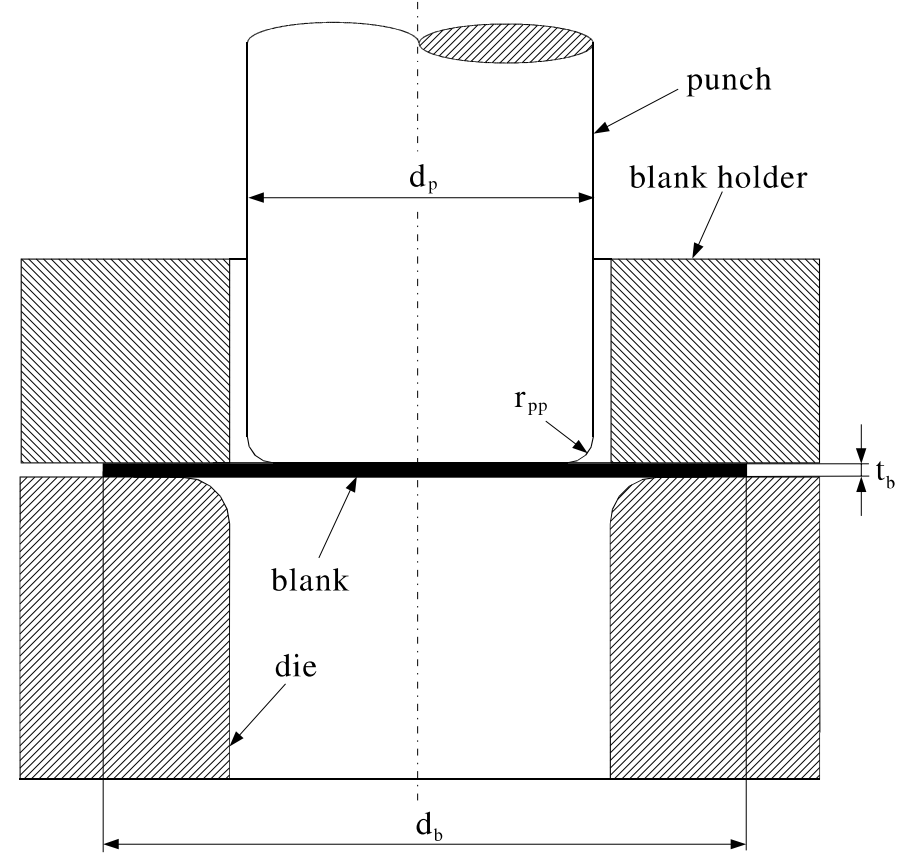

Figure 5. Geometry of the deep drawing process.

Engler and Kalz (2004) the relative earing heights are documented. Therefore, the normalized earing profile is discussed here. It can be seen that the discrete model overestimates the earing height. This means that the anisotropy is overestimated. The continuous model also overestimates the earing height if the half-width is chosen in order to fit the experimental codf. If the half-width $b$ is enlarged by a factor $\beta$ of 2 then the continuous model accurately predicts the earing profile. The modification of the half-width allows us to correct the predictions of the Taylor model which inherently overestimates the sharpness of the texture. In the next example we will modify the model DT such that the significant overestimation of anisotropy is avoided.

\subsection{Example: modification of the discrete model}

The second example has confirmed the fact that the Taylor model overestimates the anisotropy of the sheet metal. This has been seen for both Taylor-type models (DT and CT). The Taylor model based on continuous model functions allows for a correction of the predicted anisotropy by modifying the half-width while keeping the number of crystals fixed. In the case of the 


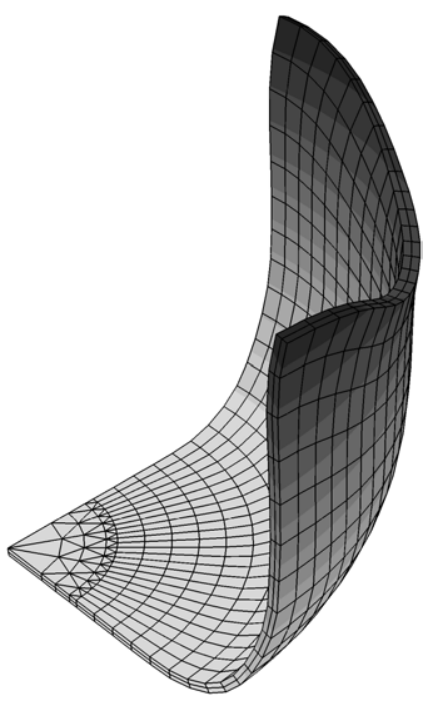

Figure 6. FE mesh of a quarter of the cup (CT model with $\beta=1$ ).

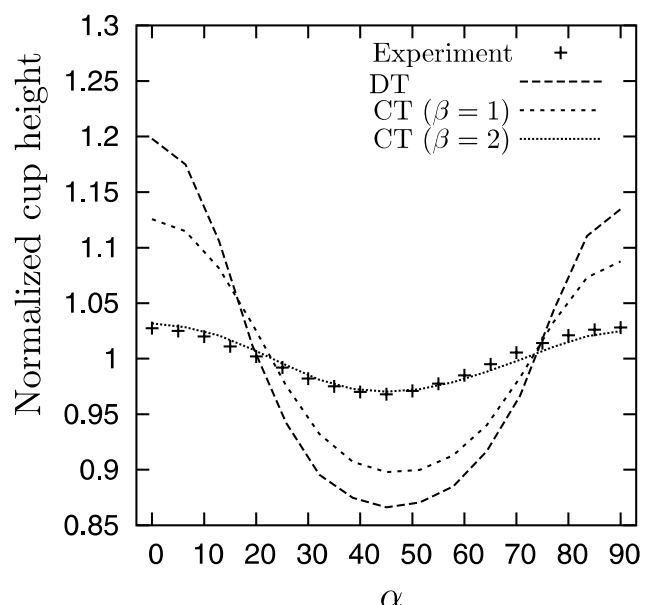

Figure 7. Comparison of the earing profile calculated by the DT and CT models with experimental data (Engler and Kalz 2004).

discrete model only an enlargement of the total number of crystals would allow for a better prediction of the earing profile. The additional crystal orientations have to approximate the scattering of orientations around the ideal texture components. The second example has also shown that the scattering should be larger than in the experimental codf due to the inherent property of the Taylor model to overpredict the anisotropy. Since more ambiguous texture based material models such as self-consistent schemes require a much higher computational effort as the Taylor-type models, it is worth looking for a simple modification of the models discussed here in order to overcome the aforementioned problem.

The isotropic or random background of the codf has been neglected so far in the DT model. The random part of the crystallographic texture induces an isotropic effective viscoplastic behaviour. The specific type of isotropic behaviour depends on the slip systems which can 


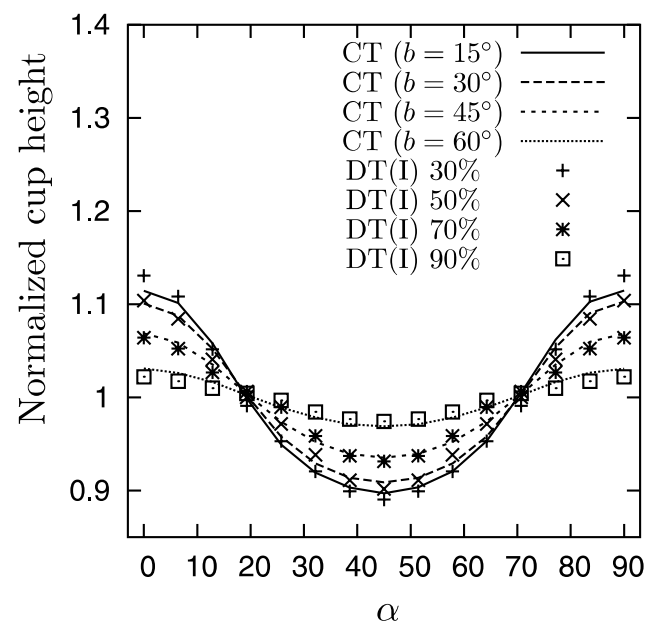

Figure 8. Earing profile for a cube texture corresponding to different isotropic volume fractions in the discrete model and different half-widths in the continuous model.

be activated in the grains. In the case of fcc materials the effective isotropic behaviour is not necessarily of the von Mises type (Böhlke 2004), but it can be assumed that the details of the isotropic behaviour are of minor importance compared with the anisotropic behaviour.

Therefore, we modify the DT model by decomposing the stress into two parts. One part describes the isotropic effective viscoplastic behaviour due to the random texture. The other part is modelled as before by the superposition of the crystal stresses. As a result, we have two types of volume fractions. One type corresponds to the single crystals, while the other describes how isotropic the microstructure is. If the volume fraction of the isotropic part is chosen to be larger than the isotropic part of the codf then the overestimation of anisotropy can be avoided.

The elasto-viscoplastic isotropic material model is specified as follows. We assume a linear and isotropic relation between the 2nd Piola-Kirchhoff stress tensor and Green's strain tensor in the unloaded configuration. The corresponding Eulerian formulation has the same form as equation (2), but the isotropy allows for the simplification $\mathbb{C}_{\mathrm{e}} \approx \tilde{\mathbb{C}}$ if the elastic strains are small. For the same reason only the evolution of $\boldsymbol{B}_{\mathrm{e}}=\boldsymbol{F}_{\mathrm{e}} \boldsymbol{F}_{\mathrm{e}}^{\top}$ has to be modelled by an evolution equation. This evolution equation is specified in a viscoplastic setting with a power law linking the equivalent von Mises stress to the equivalent viscoplastic strain rate. For brevity, the details of the formulation are not given here. The general framework can be found in the work by Simo and Miehe (1992). In the CT model an isotropic part has already been introduced. Such an approach permits varying the isotropic volume fraction independently of the half-width values. For the viscoplastic flow rule the validity of the power law will also be assumed. The material parameters are specified as follows. The exponent in the power law is taken to be identical in the isotropic and anisotropic models. The yield stress in the isotropic model is determined based on the critical resolved shear stress and the Taylor factor of 3.06.

The predictions of the modified models are now analysed in the case of a pure cube texture. In figure 8 the predicted earing profiles are shown for both models. In the case of the discrete model the volume fraction of the isotropic part has been varied in the range 30-90\%. In the case of the continuous model the half-width has been varied in the range $15^{\circ}-60^{\circ}$. It can be seen that the isotropic volume fraction of $30 \%$ corresponds approximately to a half-width of $15^{\circ}$. A volume fraction of $50 \%$ corresponds to a half-width of $30^{\circ}$. As a thumb rule, for half-width 
Table 4. Main texture components of a 2008-T4 sheet described by half widths, volume fractions, and Euler angles. Note that an orthotropic sample symmetry is assumed.

\begin{tabular}{llllll}
\hline$i$ & $b_{i}$ & $v_{i}$ & $\varphi_{1}^{i}$ & $\Phi^{i}$ & $\varphi_{2}^{i}$ \\
\hline 1 & $20.2^{\circ}$ & 0.248 & $89.12^{\circ}$ & $89.12^{\circ}$ & $358.99^{\circ}$ \\
2 & $19.2^{\circ}$ & 0.298 & $14.69^{\circ}$ & $82.20^{\circ}$ & $326.79^{\circ}$ \\
3 & $22.1^{\circ}$ & 0.153 & $26.72^{\circ}$ & $87.86^{\circ}$ & $346.14^{\circ}$ \\
4 & $11.7^{\circ}$ & 0.038 & $89.09^{\circ}$ & $88.94^{\circ}$ & $340.98^{\circ}$ \\
Random & & 0.263 & & & \\
\hline
\end{tabular}

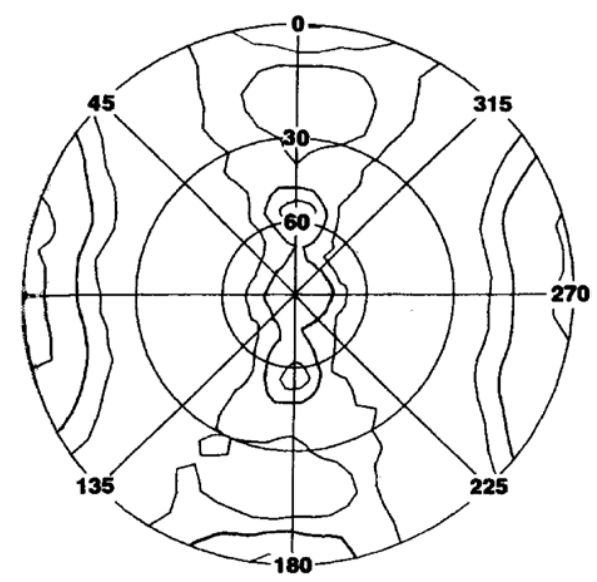

Figure 9. Experimental (100) pole figure (Lege et al 1989).

values larger than $10^{\circ}$, one can use the fact that the isotropic volume fraction in the DT model is approximately given by $(10+240 b / \pi) \%(b$ in rad). If one takes into consideration that even $b$ should be increased by a factor of 2-3 then one has a rough estimate of the isotropic volume fraction in the DT model directly based on the codf. However, this estimate depends on the number of crystals involved in the DT model. The estimate given here can be considered as an upper bound. If more discrete orientations are used, the isotropic volume fraction should be smaller. Since for a small number of crystal orientations the discrete model is computationally less expensive compared with the continuous model, the modification of the DT model seems to be versatile.

\subsection{Example: four-component texture}

Lege et al (1989) determined the volume fractions, the half-widths and the Euler angles of four texture components, which together with a random component, reproduce the main features of a crystallographic texture in a rolled aluminium sheet (see table 4). The experimental (100) pole figure is shown in figure 9. The corresponding pole figure based on the parameters given in table 4 is shown in figure 10. In figure 11 the pole figures of the four texture components are shown separately. The half-width parameters are slightly different from the values determined by Lege et al which is due to the application of different model functions for the components. Here, Mises-Fischer distribution functions are applied, whereas Lege et al used pseudoGaussian distribution functions (see, e.g. Bunge 1993). Besides the crystallographic texture, Lege et al (1989) determined the $R$ values and the yield stresses by tensile tests. 


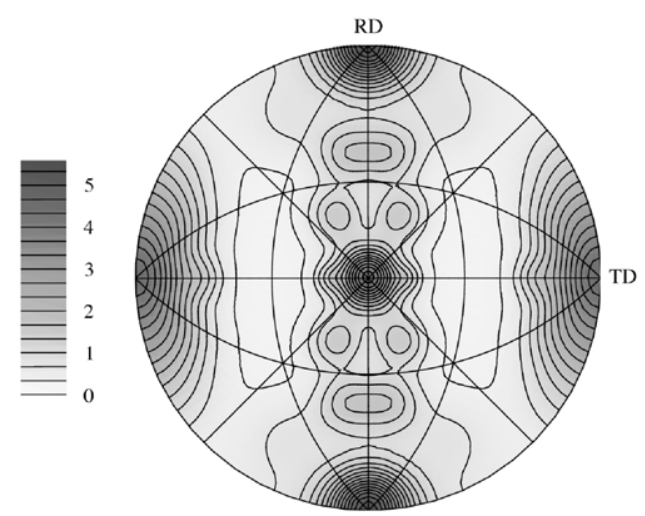

Figure 10. Approximation of the experimental (100) pole figure.

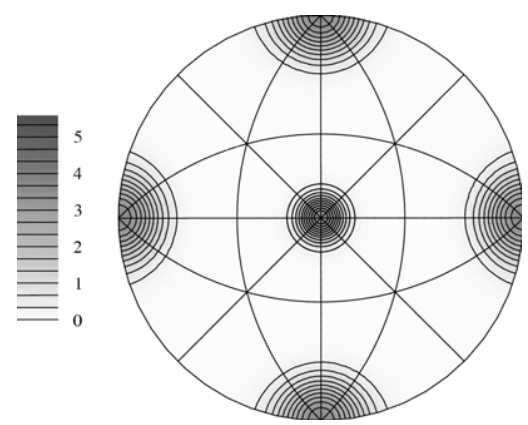

(a) Component 1

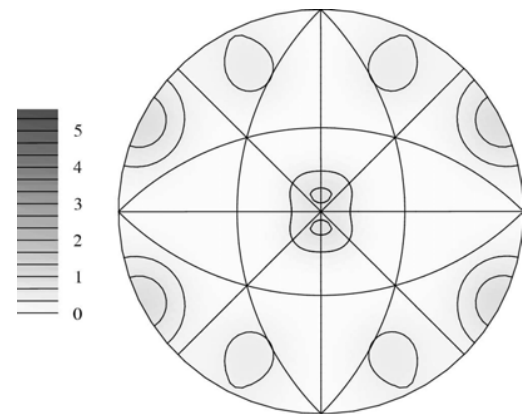

(c) Component 3

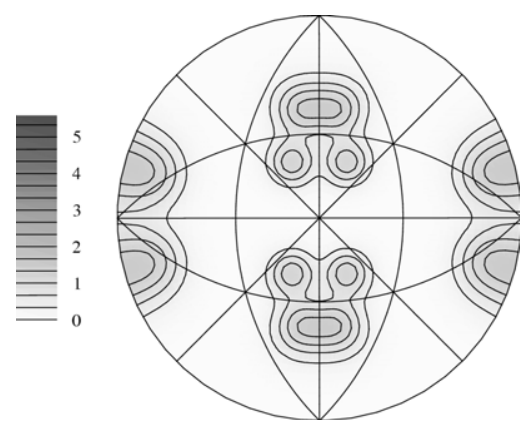

(b) Component 2

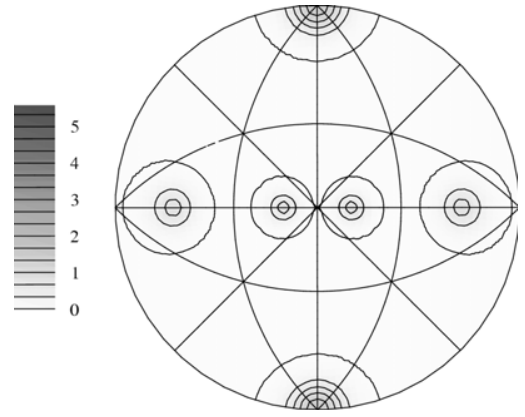

(d) Component 4

Figure 11. (100) pole figures of the texture components given in table 4 .

Figures 12 and 13 show the experimental and computed yield stresses. Figures 14 and 15 show the corresponding $R$ values. The discrete and continuous models are specified by the parameters given in table 4 .

Lege et al (1989) determined the yield stresses and the $R$ values by two different testing methods which yield slightly different results. They also computed the yield stress and the $R$ value based on Taylor-Bishop-Hill theory and also based on a model by Barlat and Lian (1989). Note that Lege et al performed the Taylor-Bishop-Hill simulation with a large number of single crystals. The Taylor-Bishop-Hill theory overestimates the 


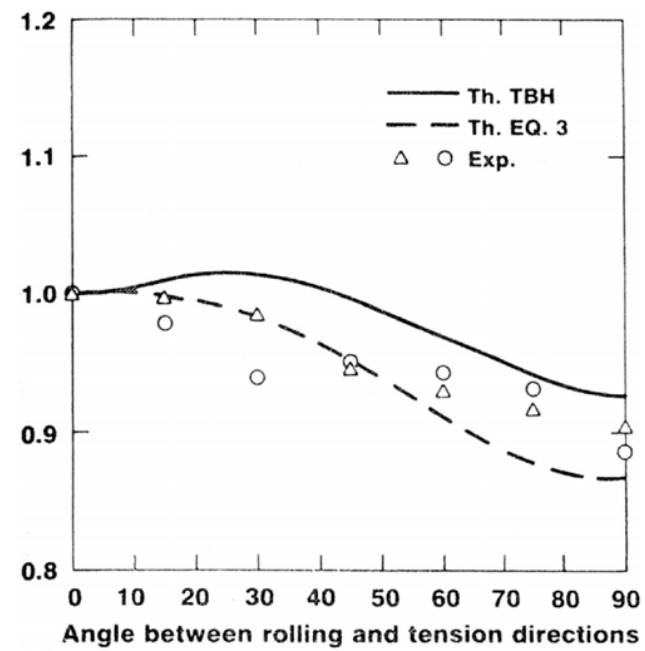

Figure 12. Normalized yield stress versus angle to the rolling direction (experimental and numerical results by Lege et al (1989).

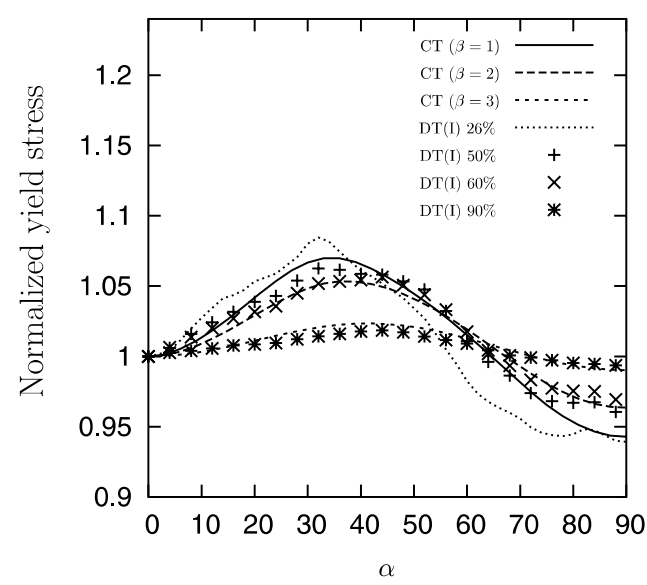

Figure 13. Normalized yield stress versus angle to the rolling direction for the discrete and the CT model.

relative yield stress and predicts a maximum which cannot be found in the experimental data. Our texture simulations show the same trend (see figure 13). The simulations with the discrete model have been performed with isotropic volume fractions $26 \%, 50 \%, 60 \%, 90 \%$, respectively. For the simulations with the continuous model the $\beta$ values 1,2 and 3 have been used. Neither of the Taylor models is able to predict the directional dependence of the yield stress qualitatively. This corresponds to the findings of Lege et al. Since the same homogenization scheme is applied, it cannot be expected that the predictions are better in our case. Note that Lege et al did not combine the Taylor-Bishop-Hill estimate with an FE simulation.

In contrast to the yield stress, the predicted $R$ values correspond better to the experimental findings. Furthermore, the numerical results indicate that the discrete model overestimates the anisotropy much more than the continuous model. As a result, the variation in $R$ with $\alpha$ is 


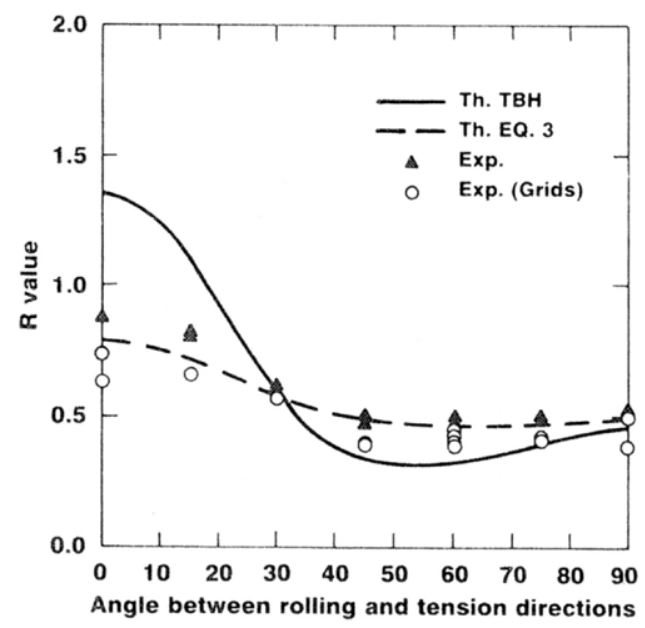

Figure 14. $R$ value versus angle to the rolling direction (experimental and numerical results by Lege et al (1989).

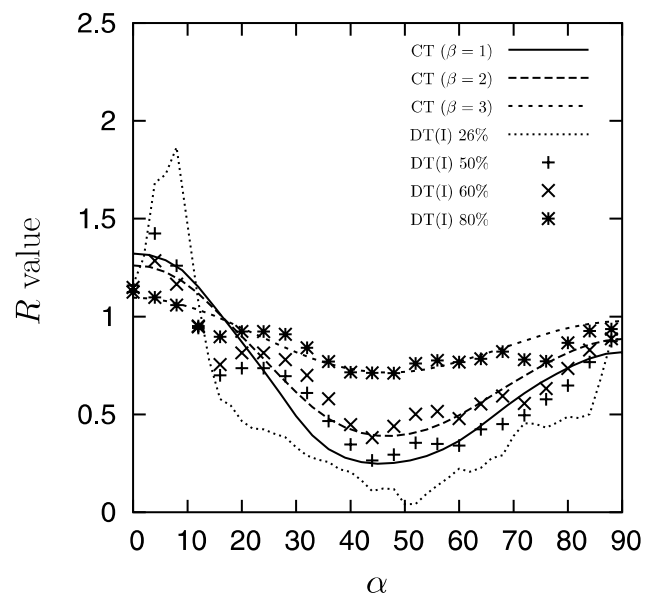

Figure 15. $R$ value versus angle to the rolling direction for the discrete and the CT model.

much less pronounced compared with our predictions based on the discrete model with four components. If one compares the numerical $R$ values with numerical yield stress then it can be seen that the $R$ value depends sensitively on the number of crystals. If the isotropic volume fraction in the discrete model and the half-width in the continuous model are increased, the $R$ value is smoothed out in both cases as already discussed in the last example.

The geometrical parameters for the simulation of the deep drawing process are the following: $d_{\mathrm{b}}=162 \mathrm{~mm}, d_{\mathrm{p}}=97 \mathrm{~mm}, t_{\mathrm{b}}=1.24 \mathrm{~mm}, r_{\mathrm{pp}}=4.95 \mathrm{~mm}$ (Lege et al 1989). A quarter of the sheet has been discretized by $960 \mathrm{C} 3 \mathrm{D} 8 \mathrm{H}$ and $132 \mathrm{C} 3 \mathrm{D} 6 \mathrm{H}$ elements. In figure 16 the normalized earing profiles calculated by the continuous model for different half-width parameters are shown. If the half-widths are increased by a factor of 3 then there is good agreement of the predictions of the continuous model with the experimental results. If an isotropic volume fraction of $90 \%$ is used then the DT model predicts the experimental results (see figure 17). Figure 18 shows a comparison of the earing profiles 


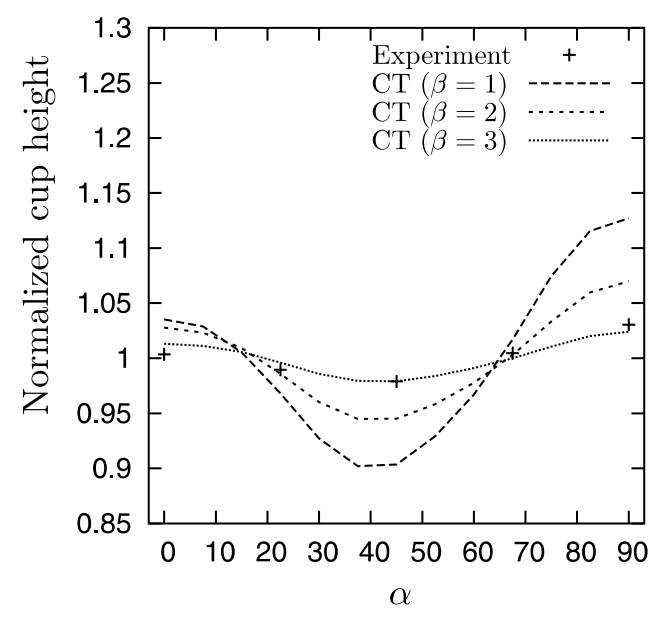

Figure 16. Comparison of the earing profiles calculated by the continuous model for different half widths and based on experimental data by Lege et al (1989).

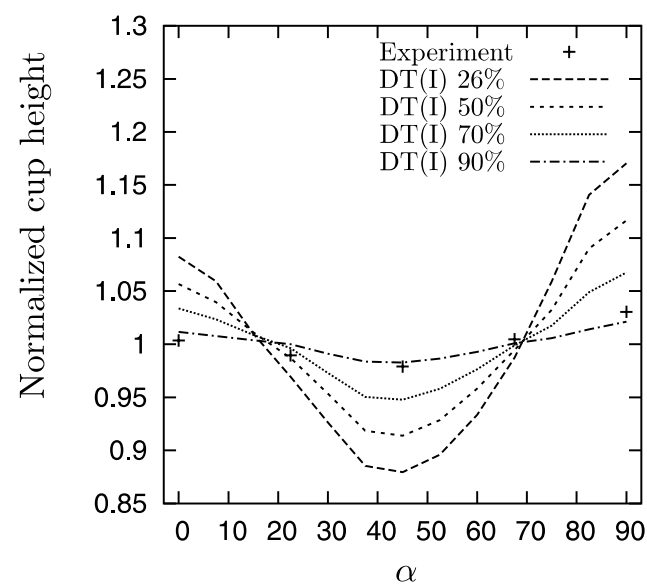

Figure 17. Comparison of the earing profiles calculated by the discrete model for different isotropic volume fractions and based on experimental data by Lege et al (1989).

calculated by the continuous and discrete models. Here it can be seen that for large half-width values (CT model) and large volume fractions (DT model), the solutions for both models are similar.

\section{Summary and conclusions}

In the present paper two Taylor models have been used to simulate the mechanical behaviour of aluminium under large plastic deformations. One Taylor model (DT) is based on a discrete representation of the codf. The other Taylor model (CT) represents the codf by a number of continuous model functions on the orientation space. The numerical examples indicate the following: (1) Both models give almost the same predictions of the viscoplastic behaviour if the DT model is applied with a large number of crystals. (2) If an approximation of the experimental codf is used to specify the orientational data of the two models then both models 


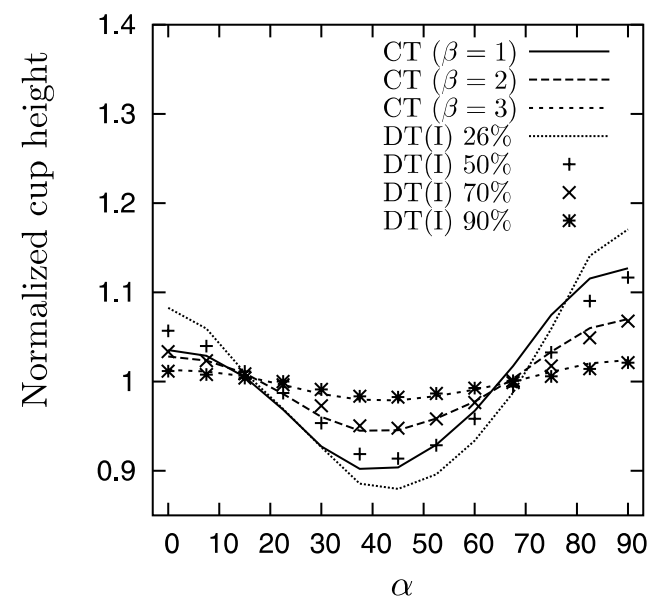

Figure 18. Comparison of the earing profiles calculated by the discrete model for different isotropic volume fractions and the continuous model for different half widths.

overestimate the plastic anisotropy. (3) In the case of the CT model this overestimation of anisotropy can be reduced by increasing the half-widths of the model functions. (4) In the case of the DT model the overestimation can be eliminated by modelling an isotropic 'single crystal' with specific volume fraction. The effective stress of the aggregate is the weighted arithmetic mean of the conventional stress tensor predicted by the Taylor model and of the isotropic material. (5) There is a simple relationship between the half-width in the continuous model and the volume fraction of the isotropic 'single crystal' in the discrete model.

\section{References}

2003 ABAQUS/Standard (Hibbitt, Karlsson and Sorensen, Inc)

Barlat F and Lian J 1989 Plastic behavior and stretchability of sheet metals: Part I. A yield function for orthotropic sheets under plane stress conditions Int. J. Plast. 5 51-66

Bertram A 1999 An alternative approach to finite plasticity based on material isomorphisms Int. J. Plast. 15 353-74 Bertram A 2005 Elasticity and Plasticity of Large Deformations (Berlin: Springer)

Bertram A and Olschewski J 1993 Zur Formulierung anisotroper linear anelastischer Stoffgleichungen mit Hilfe einer Projektionsmethode Z. Angew. Math. Mech. 73 T401-T403

Boender C, Rinnooy Kan A, Timmer G and Stougie L 1982 A stochastic method for global optimization Math. Program. 22 125-40

Böhlke T 2004 The Voigt bound of the stress potential of isotropic viscoplastic fcc polycrystals Arch. Mech. 56 423-43

Böhlke T 2005 Application of the maximum entropy method in texture analysis Comput. Mate. Sci. 32 276-83

Böhlke T and Bertram A 2001 The evolution of Hooke's law due to texture development in polycrystals Int. J. Solids Struct. 38 9437-59

Böhlke T, Bertram A and Krempl E 2003 Modeling of deformation induced anisotropy in free-end torsion Int. J. Plast. 19 1867-84

Brandes E and Brook G 1998 Smithells Metals Reference Book 7edn (Oxford: Butterworth-Heinemann)

Bronkhorst C, Kalidindi S and Anand L 1992 Polycrystalline plasticity and the evolution of crystallographic texture in fcc metals Phil. Trans. R. Soc. Lond. A $341443-77$

Bunge H-J 1993 Texture Analysis in Material Science (Göttingen: Cuviller Verlag)

Csendes T, Daroczy B and Hantos Z 1986 Nonlinear parameter estimation by global optimization: comparison of local search methods in respiratory system modelling System Modelling and Optimization: Proceedings of the 12th IFIP Conference (Berlin: Springer-Verlag) p 188-92

Engler O and Kalz S 2004 Simulation of earing profiles from texture data by means of a visco-plastic self-consistent polycrystal plasticity approach Mater. Sci. Eng. A 373 350-62

Eschner T 1993 Texture analysis by means of model functions Texture. and Microstruct. 21 139-46 
Eschner T and Fundenberger J 1997 Application of anisotropic texture components Texture. Microstruct. 28 181-95 Hutchinson J 1976 Bounds and self-consistent estimates for creep of polycrystalline materials Proc. R. Soc. Lond. A 348 101-27

Kocks U 1976 Laws for work hardening and low temperature creep J. Eng. Mater. Technol. (ASMEH) $9875-85$

Kocks U and Mecking H 2003 Physics and phenomenology of strain hardening: the FCC case Progn. Mater. Sci. 48 $171-273$

Kocks U, Tome C and Wenk H 1998 Texture and Anisotropy: Preferred Orientations in Polycrystals and Their Effect on Materials Properties (Cambridge: Cambridge University Press)

Lege D, Barlat F and Brem J 1989 Characterization and modelling of the mechanical behavior and formability of a 2008-T4 sheet sample Int. J. Mech. Sci. 31 549-63

Les P, Zehetbauer M, Rauch E and Kopacz I 1999 Cold work hardening of Al from shear deformation up to large strains. Scr. Mater. 41 523-8

Mandel J 1974 Thermodynamics and plasticity Proc. Int. Symp. Foundations of Continuum Thermodynamics ed J D Domingos et al (London: McMillan) p 283

Mardia K and Jupp P 2000 Directional Statistics (New York: Wiley)

Mathur K and Dawson P 1989 On modeling the development of crystallographic texture in bulk forming processes Int. J. Plast. 5 67-94

Matthies S 1980 Standard functions in texture analysis Phys. Status Solidi. b 101 K111-15

Matthies S, Muller J and Vinel G 1988 On the normal distribution in the orientation space Texture Microstruct. 10 77-96

Mecking H 2001 Work hardening of single-phase polycrystals Encyclopedia of Materials: Science and Technology (Amsterdam: Elsevier Science) pp 9785-95

Miehe C, Schröder J and Schotte J 1999 Computational homogenization in finite plasticity. Simulation of texture development in polycrystalline materials Comput. Methods Appl. Mech. Eng. 171 387-418

Molinari A, Canova G and Ahzi S 1987 A self consistent approach of the large deformation polycrystal viscoplasticity Acta Metall. 352983

Ogden R 1972 Large deformation isotropic elasticity: on the correlation of theory and experiment for compressible rubberlike materials Proc. R. Soc. Lond. A 328 567-83

Raabe D and Roters F 2004 Using texture components in crystal plasticity finite element simulations Int. J. Plast. 20 339-61

Raabe D, Klose P, Engl B, Imlau K-P, Friedel F and Roters F 2002 Concepts for integrating plastic anisotropy into metal forming simulations Adv. Eng. Mater. 4 169-80

Rychlewski J and Zhang J 1989 Anisotropy degree of elastic materials Arch. Mech. 47 697-715

Schaeben H 1992 'Normal' orientation distribution Texture. Microstruct. 19 197-202

Schaeben H 1994 Diskrete mathematische Methoden zur Berechnung und Interpretation von kristallographischen Orientierungsdichten DGM Informationsgesellschaft mbH

Simo J and Miehe C 1992 Associative coupled thermoplasticity at finite stains: formulation, numerical analyses and implementation Comput. Methods Appl. Mech. Eng. 98 41-104

Taylor G 1938 Plastic strain in metals J. Inst. Met. 62 307-24

Truesdell C and Noll W 1965 The Non-Linear Field Theories of Mechanics Encyclopedia of Physics vol III/3 (Berlin: Springer)

van Houtte P 1988 A comprehensive mathematical formulation of an extended Taylor-Bishop-Hill model featuring relaxed constraints, the Renouard-Winterberger theory and a strain rate sensitive model Texture. Microstruct. 8-9 313-50

Šilhavý M 1997 The Mechanics and Thermodynamics of Continuous Media (Berlin: Springer)

Wasserman G and Grewen J 1962 Texturen metallischer Werkstoffe (Berlin: Springer)

Zheng Q-S and Fu Y-B 2001 Orientation distribution functions for microstructures of heterogeneous materials: II. Crystal distribution functions and irreducible tensors restricted by various material symmetries Appl. Math. Mech. 22 885-903 